\title{
El hombre detrás de la obra: vida y obra del Obispo Enrique Chávez Campos en el Centenario de su nacimiento, fundador de la Iglesia Pentecostal de Chile
}

Oscar Corvalán $V^{1}$

\section{RESUMO}

Este artigo apresenta a biografia bispo Enrique Chávez Campos, fundador da Igreja Pentecostal do Chile. Ele foi um homem que se esforçou durante toda a sua vida para superar vários obstáculos e limitações que se lhe apresentaram para chegar a ser um líder Pentecostal nacional e latino-americano. A resiliencia mostrada durante toda a sua vida indica sua capacidade para vencer a muitos problemas, sempre tendo em mente uma visão clara para onde deveria caminhar. É também importante conhecer suas posturas de abertura ecumênica e lutas pela unidade dos evangélicos em nível nacional e continental.

\section{PALAVRAS-CHAVE}

Líderes Pentecostais Chilenos, resiliencia Pentecostal, Igreja Pentecostal do Chile.

\section{ABSTRACT}

This paper presents the biography of the Bishop Enrique Chávez Campos, founder of Pentecostal Church of Chile. Bishop

\footnotetext{
${ }^{1}$ Oscar Corvalán V., PhD. (Sociólogo e doutor em Educação), participa do Foro Cristiano Global e é secretario da equipe pastoral encarregada da organização do Foro Pentecostal Latinoamericano y del Caribe.
} 
Enrique Chávez Campos endured all his life in order to overcome obstacles and limitations before him in order to become a National and Latin American Pentecostal Leader. The resiliency he showed in his life indicates his ability to win several problems/ troubles, always with a clear vision of the way to be pursued. It is also important to know his Ecumenical perspectives and his fights for the evangelical unity both in National as in Continental perspectives.

\section{KEY-WORDS}

Pentecostals Chilean Leaders, Pentecostal resiliency, Pentecostal Church of Chile.

\section{Introducción}

Al cumplirse cien años de su nacimiento y sobre la base de dos entrevistas concedidas por la hija menor del obispo Enrique Chávez Campos, Rosita Chávez Reyes el 10 de Febrero y el 1ro. Mayo de 2012, en su casa de la ciudad de Curicó y rodeada de hijas y nieto, así como conversaciones sostenidas con su único hijo, Manuel Chávez Campos y fotografías aportadas por la primera, ha sido posible reconstruir una primera biografía de la vida del citado obispo.

Del análisis de los datos aportados por sus hijos y el testimonio de los pastores que trabajaron con él, diez de los cuales fueron entrevistados por el autor, resulta evidente que se trató de un hombre que se esforzó durante toda su vida por superar cientos de obstáculos y limitaciones que se le presentaron para llegar a ser el líder Pentecostal nacional y latinoamericano que muchos tuvieron la oportunidad de conocer. A menudo se tiene la idea que quienes llegan a legar una gran obra en una comunidad determinada son super hombres, y, por tanto, dotados de una energía, fuerza y espíritu privilegiados a la que no pueden aspirar la mayoría de los mortales. Pero la resiliencia mostrada durante su vida por quien llegara a ser el obispo Enrique Chávez, muestra más bien su capacidad para sobreponerse a multitudes 
de problemas, teniendo siempre en mente una visión clara de hacia donde avanzar. ${ }^{2}$

De particular relevancia resulta conocer sus posturas de apertura ecuménica y luchas por la unidad de los evangélicos a nivel nacional y continental. Sin embargo, en este informe nos proponemos conocer al ser humano que existió detrás del líder Pentecostal destacado.

El relato que sigue está construido sobre la base de un eje cronológico, pero también destacando algunos de los hitos más importantes de la vida del Obispo Enrique Chávez Campos, los que permiten visualizar mejor las motivaciones, actitud persistente y continuidad de la visión y misión que se fijó este personaje cristiano Pentecostal, ya que ambos aspectos resaltan paralelamente en su obra. Se podría decir que nunca tuvo una actitud de Pentecostal sectario que pudiera considerarse especial y dueño de la verdad, sino que el perfil de una persona dialogante, dispuesta a someter a prueba sus aseveraciones, acogedor de las propuestas divergentes, pero firme en su propósito de construir una estructura eclesiástica que acogiera al máximo de personas posible. Para una mejor comprensión de lo que fue la vida del pastor Enrique Chávez Campos en Curicó es preciso imaginarse lo que fue su vida previa en Santiago, porque fue precisamente en ese periodo en que se templó su carácter y en que se preparó para la misión que desarrollará como adulto en y desde Curicó, Chile.

Los siguientes dos apartados de este capítulo, en apretada síntesis, perfilan un cuadro del carácter y personalidad del joven Enrique Chávez Campos, así como resumen la obra de este pastor fundador de una de las denominaciones pentecostales chilenas caracterizadas por su apertura al diálogo y a la unidad de todos los cristianos que confiesan que Jesucristo es el Señor.

El autor de estas líneas se ha preguntado repetidamente sobre el significado de las enseñanzas del fundador de la Iglesia Pentecostal de Chile para la misma, sus miembros, sus pastores, sus autoridades, su

\footnotetext{
${ }^{2}$ Luis Cruz Villalobos. "Resiliencia y Experiencia Pentecostal. En Mansilla, M. A. y [Orellana, L. (eds.) La Religion en Chile del Bicentenario. RELEP Ediciones. Concepción, 2011, pp. 59-78.
} 
estructura eclesial, su transparencia organizacional, sus líderes jóvenes, y sobre el impacto de la iglesia y sus miembros en este siglo XXI, luego de un centenar de pentecostalismo en el país, en especial luego de analizar que los evangélicos han pasado de menos del 1 por ciento a cerca del 20 por ciento de la población chilena. Precisamente ahora que muchas comunidades Pentecostales a menudo han dejado lugar a celebrar el evento, incluso el espectáculo y la entretención, llenos de promesas de prosperidad predicada, en medio de una sociedad que ha superado la extrema pobreza original y que busca en los bienes tangibles lo que los primeros pentecostales buscaron en la Espíritualidad Pentecostal.

Si el lector de estos párrafos logra hacer la relación entre la vida del fundador de esta Iglesia, su contexto socio-político-económico y religioso y su época actual y condición personal, que lo lleve a una reflexión sobre su propia práctica personal y vida espiritual; entonces, se cumplirá el propósito de haber tratado de organizar estos párrafos sobre la vida - sin duda ejemplar - de este cristiano y Pentecostal al mismo tiempo, que se llamó Enrique Chávez Campos.

\section{La vida en Santiago del hermano Enrique Chávez Campos}

La vida de militante cristiano la comenzó el Obispo Chávez hacia los 12 años de edad, cuando, proveniente de una familia popular católica, se convierte al evangelio en la Iglesia Metodista Pentecostal ubicada en calle Jotabeche 40 de Santiago, hoy comuna de Estación Central. Su permanente búsqueda espiritual lo llevó a relacionarse primeramente solo, sin ningún miembro de su familia de origen, con dicha iglesia Pentecostal, pero con el correr del tiempo lo acompañó su madre, doña Rosa Campos.

La vida de niñez y adolescencia de Enrique Chávez Campos sólo llegó hasta los 14 años porque a esa edad su padre, don Erasmo, emigró a trabajar en las minas del norte y nunca más volvió. Como consecuencia de ello, a esa edad comienza a trabajar y estudiar paralelamente, para poder sostener a sus 5 hermanos menores. El abandono total del padre de la familia debe haberle producido un impacto psico-afectivo 
tan grande que decidió quitarse su segundo nombre, ya que había sido bautizado como Enrique Erasmo.

Corrían entonces en Chile los años de la segunda mitad de los años 1920s, caracterizados por la convulsión social experimentada por el Gobierno de Arturo Alessandri Palma, durante los cuales se produce la revuelta de las clases populares lideradas por los mineros del norte del país y manifestada en la ciudad capital del país por manifestaciones sociales diversas que protestaban por la falta de trabajo y educación, viviendas insalubres, y un clima político propenso a periódicos estallidos sociales de los trabajadores demandando justicia social.

La educación pública era extremadamente escasa durante los años de niñez y adolescencia del joven Enrique. Chile tuvo que esperar hasta 1930 para llegar a completar 12.650 docentes y para-docentes. Por aquellos años, la gran mayoría de la población del país era analfabeta y escaseaban por todo el territorio las escuelas públicas. Hacia el año 1895 la matrícula total llegaba a 150.000 alumnos repartidos en 1.300 pequeños centros educativos fiscales. No fue sino hasta mediados de los años 1960 que se logró triplicar estas cifras (Correa, S. et al. 2001:24), lo cual se refleja en la mayoría de adultos analfabetos hasta después durante todo el primer siglo de la independencia de Chile, según se desprende del cuadro $\mathrm{N}^{\mathrm{o}} 1$.

\section{Cuadro $\mathbf{N}^{0} 1$}

Chile: evolución de las tasas de analfabetismo 1854-1930.

\begin{tabular}{|c|c|c|c|c|c|c|c|c|}
\hline Año & 1854 & 1865 & 1875 & 1885 & 1895 & 1907 & 1920 & 1930 \\
\hline Porcent. & 86.5 & 83.0 & 77.1 & 71.1 & 68.2 & 60.0 & 50.0 & 44.0 \\
\hline
\end{tabular}

Fuente: Labarca H., Amanda. 1939:276.

Los mejores índices de retención en primaria los muestra recién en el año1950 la provincia de Santiago de Chile con un 53\% en el sexto grado urbano y un $22 \%$ en escuelas rurales (Havighurst, Robert, J. 1962:185). Cuando el pastor Enrique Chávez Campos organiza la Iglesia Pentecostal de Chile, en 1947, casi un tercio de los adultos eran 
analfabetos y de 900.000 niños en edad escolar, casi la mitad no asistían a la escuela. La mortalidad infantil era desastrosa, ya que un estudio hecho por un ministro de Salud Pública de la época mostró que el 50 por ciento de los niños morían antes de llegar a los nueve años (Correa, S., et al. 2001:188).

Es en este contexto de falta de educación y salud pública, de alto desempleo, de falta de oportunidades de todo tipo que se templa el carácter del joven Enrique, en los sectores populares de Santiago llenos de conventillos insalubres, de necesidades de todo tipo, de alto alcoholismo y prostitución entre los sectores populares que luchaban contra la tuberculosis y las enfermedades físicas, psicológicas y sociales.

Durante la segunda mitad del primer siglo después de la independencia de Chile (S. XX) y hasta el año 1938, en el país se libraron enconadas batallas entre educadores liberales partidarios de la educación pública, por un lado, y conservadores apoyados por la iglesia católica, por el otro. Ello se centró en los contenidos del currículum, especialmente en la lucha por reemplazar el latín por una mayor dedicación al estudio de las ciencias y las técnicas comerciales e industriales, así como para que la educación obrara como un agente fundamental en el afianzamiento e integración de la nación. Interminables discusiones entre educadores y políticos, y entre autoridades del Estado y eclesiásticas, explican en parte por qué la Ley de Instrucción Primaria Obligatoria de 1920 en Chile no fuese promulgada sino hasta 1938 (Correa, S. 2001:40-41). A partir de ese periodo, el número de alumnos matriculados en la educación primaria pasó a 500.000 en 1925, a 795.600 en 1950. En dicho periodo, la educación secundaria aumentó su matrícula de 63.500 a 148.900 alumnos, mientras que la matrícula universitaria casi se duplicó, pasando de 6.200 a 11.000 estudiantes. Por lo tanto, la construcción de un verdadero sistema de educación pública debió esperar más de un siglo de independencia para establecerse a nivel nacional, posibilitando al menos el acceso del alumnado urbano, ya que la red de centros educativos rurales recién se vino a establecer en la segunda mitad del siglo XX.

Estas circunstancias explican en buena parte el por qué nunca se vió en la oficina del obispo Chávez un diploma de graduación alguna. En efecto, él fue un gran autodidacta que muy tempranamente comprendió 
que el autoaprendizaje es una herramienta fundamental en las personas. Comenzó leyendo y analizando a fondo la Biblia para continuar con cuanto libro encontró que pudiera explicar la construcción histórico-lingüística de la misma y como Dios había guiado a una cuarentena de escritores para fuese escrita durante un periodo histórico de cerca de quince siglos que culminan en el siglo primero de la era cristiana.

En particular, la vida del joven Enrique fue extremadamente difícil por las condiciones sociales y familiares imperantes durante su adolescencia y juventud. A pesar de ello, hizo estudios vespertinos y mantenía a sus 4 hermanos (entre los que se cuentan Roberto y René, el menor), a su hermana Aurora, engendrados por don Erasmo Chávez y a su propia madre doña Rosa Campos. Con posterioridad al abandono de su esposo Erasmo, doña Rosa se casó con don Luis Álvarez, con quien tuvo dos hijos más.

De los relatos escuchados en el hogar de parte de sus padres, Rosita y Manuel destacan que la familia de origen de su padre fue muy pobre y que para construir y organizar la Iglesia Pentecostal de Chile tuve que enfrentar un sinnúmero de dificultades, entre las que se destacan acusaciones infundadas de los más graves delitos sociales y personales de la época, como fueron las acusaciones por la prensa local de ser militante comunista y usar indebidamente los fondos de la iglesia.

Don Enrique tuvo una sola hermana, doña Aurora Chávez Campos, pero en total fueron ocho hermanos, ya que aparte de los hijos de su padre don Erasmo Chávez, doña Rosa tuvo dos hijos con su segundo esposo, quienes no comparten el apellido Chávez Campos. Entre estos últimos, los hijos sobrevivientes destacan que don René Chávez Campos (menor que don Enrique) trabajó en la Policía de Investigaciones y falleció a los 89 años de edad. Es decir, su madre tuvo primero seis hijos con don Erasmo y luego dos con don Luis Álvarez. En esas circunstancias, al joven Enrique cuando desde su adolescencia le tocó trabajar de joven para educar mantener a sus hermanos.

No obstante su trabajo y lo demandante que eran las tareas asignadas al joven Enrique por su pastor Manuel Umaña Salinas, se las ingeniaba para completar en horario vespertino su educación secundaria y luego ingresó al conservatorio a estudiar guitarra y posteriormente llegó a participar en una opera y aprendió otros instrumentos, piano, acorde- 
ón y armonio. Con ello evidenciaba una personalidad extremadamente inquieta que le llevaba a estar siempre activo, creando nuevos proyectos de vida y para la extensión del Evangelio en Chile.

Sus hijos recuerdan que don Enrique contaba repetidamente la historia de una anciana que fue a la cárcel y escuchó los disparos de fusilamiento de uno de hijos, pero ella era nada menos que su madre, quien acompañó hasta el último minuto a uno de sus hijos, quien, siendo militar, muere fusilado por una calumnia que le condenó por supuesto involucramiento personal con la mujer de uno de sus superiores. Así, en algún momento deslizó que el caso se refería a uno de sus propios hermanos, a quien su madre ofreció un amor entrañable.

A pesar que por esos años los jóvenes chilenos acostumbraban a casarse entre los 18 y 21 años de edad, las múltiples obligaciones y proyecto de vida que atesoraba internamente don Enrique le llevó a casarse, luego de casi nueve años de relación afectiva, en el año 1937, con doña Anita Reyes Díaz, quien a la sazón tenía 26 años de edad. Anita era también miembro de la iglesia metodista Pentecostal, trabajaba en industria textil Caffarena desde los 15 años, como costurera fina, quien vivía con familiares en el sector Vivaceta-Gamero Club Hípico de Santiago, pero había nacido en la comuna Centro Sur de San Vicente de Tagua Tagua.

Entre las funciones que desempeñó Enrique Chávez Campos en la Iglesia Metodista Pentecostal de Chile, IMPCh, de calle Jotabeche (40), se destacó como Jefe de jóvenes, del coro, y encargado de predicación a la cárcel pública. Pero también se destacó por haber sido por años el ayudante del entonces pastor y luego primer obispo de esa iglesia don Manuel Umaña Salinas.

\section{La vida del pastor Enrique Chávez Campos en Curicó}

Con posterioridad a haber pasado muy joven por los principales cargos en la iglesia de Jotabeche, en 1939 fue enviado a hacerse cargo de la congregación de Curicó y en el año 1940 fue ungido pastor, a los 29 años de edad. Llegó a Curicó con su hija Enriqueta de un año; 
luego nacen en esta ciudad Manuel en 1940 y en 1952 su hija menor, Rosa. Pero luego de mostrar durante casi seis años sus capacidades de liderazgo y hacer crecer la iglesia formando pequeñas congregaciones en los principales villorrios y comunas de la Provincia de Curicó, sobrevino un conflicto serio con las autoridades de la IMPCh. Luego de haber criticado públicamente la poca transparencia de las finanzas en una conferencia de dicha denominación en el año 1945, fue expulsado de la misma. Según declara su hija menor Rosa, "lo desaforaron de la IMPCh porque comenzó a pedir rendición de cuentas".

De acuerdo con el testimonio de su hija menor, Rosy Chávez Reyes, al llegar a Curicó el pastor Chávez había una iglesia formada, y él comenzó a extenderla abriendo locales, a partir del cerro Condell y posteriormente el de la calle Colón casi al llegar al antigua vía férrea, actual camino a Licantén por el sector Bombero Garrido. Como la congregación era pequeña, para el sustento propio y de su familiar, cuando llegó a Curicó se instaló en cale Peña casi merced con una heladería artesanal y frutería junto con su esposa pastora Anita, pero al no tener tiempo suficiente abandonó este negocio y mas adelante se dedicó a la compra y venta de propiedades antigua que reformaba para vender.

Luego de la expulsión, el pastor Chávez se comunicó personalmente con cada uno de los principales miembros de la congregación de Curicó y les instó a quedarse en la IMPCh de esa ciudad, pero la mayoría de ellos prefirió seguir su plan de instalarse en una calle paralela y le apoyó a fundar la Iglesia Pentecostal de Chile, IPCh, a partir del año 1946, que obtuvo su personalidad jurídica el 3 de agosto de 1947.

Pero paralelamente al proceso de organización de la nueva iglesia comenzó una verdadera guerra sucia contra la misma de parte de autoridades y miembros de la IMPCh. Luego lo acusaron de comunista por los diarios y lo amenazaron con deportarlo, ya que en esa época se perseguía por parte del Gobierno a todos los sindicados de comunista, como se puede deducir del análisis de la prensa de la época. En esas circunstancias su madre doña Rosa se trastornó por las acusaciones y peligro de su hijo. Estas acusaciones incluso se pueden verificar en archivos del diario La Prensa de Curicó de la época.

Se da un cierto paralelo entre los inicios del pentecostalismo en Valparaíso, con el pastor Hoover a la cabeza, y el inicio de la Iglesia 
Pentecostal de Chile en Curicó. En efecto, en ambos casos, la prensa local se encargó de desprestigiar al movimiento descalificando severamente al líder. En el primer caso, el pastor Hoover reportó ${ }^{3}$ que el diario de la época El Chileno "comenzó la publicación de un reportaje de dos a tres columnas diarias por dos semanas, lo que fue un tejido de verdar y mentira con el colorido que convenía para producir una sensación de burla y desprecio... (denunciando) la obra de un embaucador o de un loco. Gritos, desmayos y bofetadas. Escenas trágico-cómicas. .. Denuncio de la Policía. Intervención de la Justicia. El mismo ... hizo una acusación criminal contra el pastor ante el Juez del Crímen. La acusación decía, entre otras coas, que el pastor daba a la gente un brebaje que se llamaba LA SANGRE DEL CORDERO., lo que les ponía en un letargo y les hacía caer al suelo. El pastor fue citado varias veces."

El pastor Chávez seguramente había escuchado esas situaciones de parte del pastor Manuel Umaña Salinas y los primeros hermanos de la Iglesia de Jobabeche, también habrá escuchado que el Pastor Hoover respondía: "El reproche, la crítica, la burla son más saludables para nosotros que el favor y las buenas palabras. Cuando tenemos lo primero anhelamos lo segundo, ya comenzó nuestra decadencia, porque insensiblemente iremos modificándo nuestro fervor y arrojo para no ofender a los que han comenzado a alabarnos, y poco a poco caeremos en el sueño que resultará como ha resultado en todas las demás denominaciones hasta ahora" 4 Por esto, se presume que, cuando le tocó vivir directamente estas situaciones de persecución estaba espiritualmente preparado para enfrentarlas.

Pero, en 1946 salieron de la IMP un centenar de hermanos que acompañaron al entonces pastor Enrique Chávez en los inicios de la IPCh, entre los que es posible destacar (en orden alfabétido de apellidos) a: Eduardo Abrigo (futuro pastor de San Bernardo), Oscar Corvalán Corvalán (futuro pastor de Puente Alto), José Guerra (futuro pastor de Renca), Eduardo Olave (futuro pastor del sector La Castrina, colin-

\footnotetext{
${ }^{3}$ HOOVER, W. C. Historia Del Avivamiento Pentecostal en Chile. Santiago: PRESOR. CTE $(\mathrm{s} / \mathrm{n})$.

${ }^{4}$ HOOVER, W. C. Historia Del Avivamiento Pentecostal en Chile, p. 100.
} 
dante de San Miguel y del actual San Joaquín), Humberto Pavéz (futuro Jefe del Coro de Curicó), Heraclio Cordero (de Hualañé), y tantos otros que figuran como fundadores de la IPChile.

Primero en 1946 arrendaron una antigua bodega de vinos ubicada en calle Manuel Rodriguez No. 1.155, que luego compraron en 1947 y entre ese año 1954 construyen allí e inauguran la primera nave del tempo, pero con una fachada distinta a la que se agregara posteriormente con forma de una nave enterrada. Luego de inaugurar la fachada nueva, se construye e inaugura la segunda nave hacia el sector norte del predio.

Luego de una decisión del directorio de la Iglesia, el pastor superintendente fue ungido obispo en el año 1965-7. Anteriormente había sido elegido presidente del concilio evangélico de Chile en el periodo 1961-64.

En el año 1961, autorizado por el directorio de la iglesia solicita ingreso de la misma al Consejo Mundial de Iglesias, CMI, cuya sede está en Ginebra, Suiza, lo cual fupe aceptado durante la Asamblea General del organismo celebrada en New Delhi, India. Posteriormente el obispo Chávez fue elegido miembro del directorio del Consejo Latinoamericano de Iglesias, CLAI, durante asamblea constituyente celebrada en Huamapani, Lima, Peru el año 1980.

Sin embargo, esta agitada vida de dirigente nacional, regional y mundial del pentecosalismo lo lleva a Ames, Iowa, en 1985 a celebrar un pacto de hermandad con la Iglesia Unida de Cristo, de los Estados Unidos de Norteamérica, y durante el viaje cayó enfermo en junio de 1985. Posteriormente regresa a Curicó, Chile, y sufre de demencia senil, haciendo difícil la aceptación de su enfermedad mental luego de haber mostrado ser uno de los más preclaros líderes evangélicos del continente. Falleció el 24 noviembre de 1990, pero deja una obra de iglesias pentecostales esparcida por todo el territorio nacional. No obstante, lo difícil del quinquenio de su enfermedad, su presencia contribuyó a que se establecieran los liderazgos que posteriormente darían continuidad a la obra y la iglesia pudo continuar funcionando sin divisiones ni situaciones de lucha interna. Ello facilitó que a posteriori pudiera consolidarse un nuevo liderazgo en la iglesia que llevó a ungir obispo al pastor Luis Ulises Muñoz Moraga, quien hasta ahora dirige la Iglesia Pentecostal de Chile. 


\section{Organización de la obra social de las iglesias pentecostales chilenas}

Luego del terremoto que azotó a la región centro sur de Chile en el año 1960, comienza a llegar ayuda del Gobierno y los protestantes de EEUU y se organiza la institución Ayuda Cristiana Evangélica, como un Departamento del Concilio Evangélico de Chile. Como parte de esta organización, el mismo pastor Chávez se encargaba de repartir alimentos, ropa y medicamentos en Curicó. Atendía en su casa a toda hora a quien llegara o llamara por teléfono a cualquier hora, incluso en su casa se atendía a hermanas que tenían su guagua en la casa pastoral. También llegaba cada día una docena de pordioseros que pasaban a buscar almuerzo e incluso compartía su propio plato de comida. Así mismo fomentó la tradición de que hermanos de los campos enviaran frutos del país a la casa pastoral para que hubiera redistribución y consumo de quienes llegaban necesitados a la casa del obispo.

En esta época el pastor Chávez tiene la ocasión de visitar Tierra Santa y posteriormente recordaba a menudo su visita al muro de los lamentos de Jerusalem y de la manifestación espiritual que vivió al visitar la tumba del Señor Jesús. Probablemente ambas viviencias le inspiraban y otorgaban el valor espiritual para seguir adelante en la lucha por ensanchar el Reino de los Cielos en la tierra, pero también en la lucha con sus congéneres pastores y obispos por obtener un mayor reconocimiento para el pueblo Pentecostal. Algunos de sus aliados fueron el obispo y pastor Anabalón. Hospedó mucho tiempo al hijo del obispo Francisco Anabalón Duarte para reponerse de alguna enfermedad en su juventud.

Junto con apoyar que los padres miembros de su iglesia educaran a sus hijos, cuando se presentó más adelante la oportunidad de crear alguna institución educacional evangélica, el obispo Chávez mostró que siempre se interesó en la educación y que esperaba que por la vía de un pacto con la United Church of Christ o -previamente- intentando con Oral Roberts University, se llegara a crear una universidad evangélica.

Como autodidacta, el obispo Chávez cada día comenzaba yendo al escritorio. Allí y oraba y leía la Biblia y libros cristianos por al menos dos horas antes de desayunar, pero también mientras estaba comiendo o 
leyendo iba a atender a quien llegara a solicitar sus servicios. También tenía como tradición enterrar una Biblia en cada ampliación del templo de Curicó

\section{El carácter y personalidad del pastor Enrique Chávez Campos}

Nos preguntamos en primer lugar ¿Qué pueden aprender hoy los jóvenes miembros de la Iglesia Pentecostal de Chile del carácter del fundador de esta Iglesia?

A pesar que sólo pudieran conocerle por relatos de sus abuelos y o padres, la tercera y cuarta generación de hijos de miembros de esta Iglesia no tienen sino una imagen muy borrosa de lo que fueron sus inicios y de los esfuerzos por construir una estructura organizacional evangélica en tiempos de férrea oposición católica y generalizada pobreza en el país. Es en este contexto que sobresale la figura del entonces pastor Chávez para sobreponerse a toda clase de dificultades para crear una estructura de acogida de los migrantes rurales que llegaban a la ciudad de Curicó en busca de empleo y de mejores condiciones de sobrevivencia. Quienes se venían a la ciudad a hacer el servicio militar o se contrataban como adolescentes para el servicio doméstico de los hacendados con residencia en Curicó, no dudaban en quedarse en la ciudad y nunca regresaron al campo de donde provenían 5 .

La personalidad carismática del pastor y luego obispo Pentecostal Enrique Chávez no podían dejar impávido a quien tuviera la ocasión de conocerle. De hecho, había algo en su postura que se imponía por presencia. Era imposible pasar desapercibida su llegada, sus palabras, sus intervenciones precisas en reuniones de la mas diversa índole, sean de cultos espirituales, de organización de pastores, de diálogo con las autoridades civiles, militares o eclesiásticas, o de simple conversación con sus hermanos, fieles, vecinos o amigos.

\footnotetext{
${ }^{5}$ Fue precisamente el caso del futuro pastor fundador de la Iglesia Pentecostal de Chile en Puente Alto, Oscar Corvalán Corvalán y esposa Silvia Vásquez Vásquez.
} 


\section{Diez características de la personalidad del obispo Chávez}

Si fuese posible capta en un conjunto de frases el perfil de personalidad del obispo Chávez, es posible señalar que fue:

- Un autodidacta que leía cada día la Biblia y los periódicos que daban cuenta de los hechos sociales de la época.

- Un hombre de su tiempo, dialogante e interesado en todo lo que acontecía socialmente en la ciudad, en los campos y en el país.

- Una persona interesada en el dolor humano y en buscar todas las formas posibles para aliviarlo, empezando por la restauración espiritual del ser humano, se a través de la conversión que significa un cambio de estado (metanoia, como nos lo enseñara), sea a través de ordenar las finanzas del hogar para progresar, o ambas, por supuesto.

- Alguien que estaba genuinamente interesado cuando conversaba con otra persona, manifestando una empatía infinita para sufrir y llorar con el sufriente, como también para disfrutar del gozo producido por el agradecimiento derivado de los beneficios de satisfacción personal producidos por la vida normada por seguir las enseñanzas del Maestro.

- Un padre de familia protector de la suya y de la familia extendida que para él significaba su iglesia, aún cuando sus propios hijos no pudieran disfrutar a menudo de su tiempo por considerar tan importante su familia extendida como su familia de procreación.

- Un líder Pentecostal que no tuvo complejos para relacionarse con representantes de iglesias protestantes históricas o del catolicismo reinante en el país, sino que con una actitud transparente buscó que sus seguidores pudieran también participar de todas las instancias posibles de manifestación de la comunión cristiana.

- Un pastor que buscó la comunión dentro y fuera de su iglesia, basada en la transparencia la franqueza, en la verdad y en la proyección a futuro, olvidando o perdonando rencillas y rencores pasados.

- Un predicador incansable, que, además, tenía dotes de actor y cantante, lo que hacía inolvidables sus sermones, enseñanzas e himnos que enseñaba.

- Un líder latinoamericano, quien, a pesar de sus limitaciones idiomáticas, se esforzó por dar a conocer el carácter de la iglesia evangélica y 
Pentecostal chilena en foros latinoamericanos y mundiales.

- Un amigo de confianza en quien era posible confiar los secretos del alma y de la vida, y de quien se podía esperar un consejo desinteresado, un análisis certero de la situación y una recomendación precisa de las opciones a enfrentar frente a los desafíos de la vida.

\section{El legado de la obra del Obispo Enrique Chávez Campos}

La síntesis anterior conduce directamente a analizar brevemente parte de la obra que llevó a cabo tan insigne personaje. Sin duda, la primera que salta a la vista fue ¿Cómo llegó a organizar una Iglesia Pentecostal que en su momento llegó a ser la tercera fuerza denominacional del país luego de la Metodista Pentecostal y Evangélica Pentecostal?

De acuerdo con el testimonio de su hija menor, Rosy Chávez Reyes, la vida cotidiana giraba en torno a la preocupación constante de como extender la iglesia. El pastor Chávez lo daba todo para la iglesia. Ni siquiera compraba regalos para sus hijos para Navidad. Rara vez les compraba dulces a sus hijos. Vendió incluso una casa en santiago y otra que le donaron en El Aquelarre para poder terminar de construir el templo. Lo más importante para el pastor Chávez consistía en que muchas personas llegaran a tener una experiencia de primera mano con la transformación que operaba el bautismo del Espíritu Santo en el individuo. Cabe también consignar que el obispo Chávez tuvo dos secretarios miembros de la iglesia: los hermanos Adolfo González y Eduardo Pérez. A pesar que muchos miembros de la iglesia hubieran deseado que llegaran a ser pastores dirigentes de esta, lamentablemente por razones de salud no llegaron a serlo.

\section{Referencias bibliográficas}

CANALES, Manuel. (2011). Pentecostalismo. Grupo y autografía. En Mansilla, M. A. y Orellana, L. (eds) La Religión en Chile del Bicentenario. RELEP Ediciones. CEEP. Concepción, Chile. 
CORREA, S.; Figueroa, C.; Jocelyn-Holt, A.; Rolle, C. y Vicuña, Manuel. (2001) Historia del siglo XX chileno. Editorial Sudamérica. Santiago.

CORVALÁN, V., Oscar. (2012). Education, Citizenship and Decentralization in the Latin American Bicentenary: The Chilean Case. En Auroi, Claude y Heig, Aline. Latin American Bicentenary. Dreams and Legacy. Imperial College Press. London.

CORVALÁN, V., Oscar. (2010) Distribución, crecimiento y discriminación de los evangélicos pentecostales. Revista de Estudios Teológicos y Pastorales. Comunidad Teológica Evangélica de Chile, CTE. Año, 12 No 18.II semestre de 2009 y I semestre de 2010.

CORVALÁN, V., Oscar. (2009) Pentecostalismo y Ecumenismo, en

Foro Pentecostal Latinoamericano y del Caribe, FPLC, 2012.

Cruz Villalobos, Luis (2011). Resiliencia y experiencia Pentecostal. En

Mansilla, M. A. y Orellana, L. (eds) La Religión en Chile del Bicentenario. RELEP Ediciones. CEEP. Concepción, Chile.

Góngora, Mario. (1981) Ensayo histórico sobre la noción de Estado en Chile en los siglos XIX y XX. Santiago. Ediciones La Ciudad. Havighurst, Robert J. (1962). La sociedad y la educación en América Latina. EUDEBA. Buenos Aires.

Hernández Chávez, Alicia. (2000). México. Breve historia contemporánea. México. F.C.E.

Hoover, W. C. ( $\sin$ fecha). Historia del Avivamiento Pentecostal en

Chile. Chacón H., A. y Lagos S., H. (editores) Programa PRESOR. CTE. Santiago de Chile.

Labarca H., Amanda (1939). Historia de la enseñanza en Chile. Imprenta Universitaria, Santiago de Chile.

Mansilla, M. A. y Orellana, L. (eds) La Religión en Chile del Bicentenario. RELEP Ediciones. CEEP. Concepción, Chile.

Ossenbach S., Gabriela (1993) "Estado y Educación en América Latina a partir de su independencia (siglos XIX y XX)", en Revista Iberoamericana de Educación, n. 1. enero-abril, p. 1.

Poblete M., Manuel (s/f). Historia de la Iglesia Pentecostal de Chile.

Mimeo. Curicó.

Infografía: Facebook "Yo conoci a mi obispo Chávez". 